\title{
, Structural Changes in a Single GaN Nanowire under Applied Voltage Bias
}

\author{
3 Sergey Lazarev, ${ }^{\dagger, \dagger}$ Dmitry Dzhigaev, ${ }^{\dagger}{ }^{\dagger}$ Zhaoxia Bi, ${ }^{\S}$ Ali Nowzari, ${ }^{\S}$ Young Yong Kim, ${ }^{\dagger}$ Max Rose, \\ 4 Ivan A. Zaluzhnyy, ${ }^{\dagger, \|}$ Oleg Yu. Gorobtsov, ${ }^{\dagger, \perp}$ Alexey V. Zozulya, ${ }^{\dagger, \#}$ Filip Lenrick, ${ }^{\S}$ Anders Gustafsson, ${ }^{\S}$ \\ ${ }_{s}$ Anders Mikkelsen, ${ }^{\S}$ Michael Sprung, ${ }^{\dagger}$ Lars Samuelson, ${ }^{\S}$ and Ivan A. Vartanyants*, ${ }^{\dagger}, \|_{\odot}$ \\ $6{ }^{\dagger}$ Deutsches Elektronen-Synchrotron DESY, Notkestraße 85, D-22607 Hamburg, Germany \\ $7{ }^{*}$ National Research Tomsk Polytechnic University (TPU), Lenin Avenue 30, 634050 Tomsk, Russia \\ $8{ }^{\S}$ NanoLund, Department of Physics, Lund University, P.O. Box 118, SE-221 00 Lund, Sweden \\ 9 "National Research Nuclear University MEPhI (Moscow Engineering Physics Institute), Kashirskoe shosse 31, 115409 Moscow, \\ 10 Russia
}

\section{S Supporting Information}

12 ABSTRACT: GaN nanowires (NWs) are promising building 13 blocks for future optoelectronic devices and nanoelectronics. 14 They exhibit stronger piezoelectric properties than a bulk GaN. 15 This phenomena may be crucial for applications of NWs and 16 makes their study highly important. We report on an 17 investigation of the structure evolution of a single GaN NW 18 under an applied voltage bias along polar [0001] crystallographic
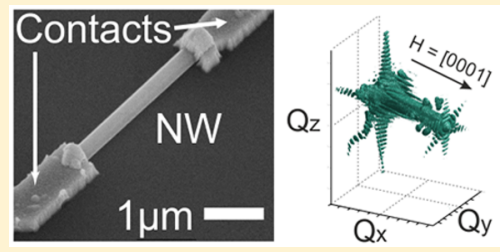
FEM model direction until its mechanical break. The structural changes were investigated using coherent X-ray Bragg diffraction. The three-dimensional (3D) intensity distributions of the NWs without metal contacts, with contacts, and under applied voltage bias in opposite polar directions were analyzed. Coherent X-ray Bragg diffraction revealed the presence of significant bending of the NWs already after metal contacts deposition, which was increased at applied voltage bias. Employing analytical simulations based on elasticity theory and a finite element method (FEM) approach, we developed a 3D model of the NW bending under applied voltage. From this model and our experimental data, we determined the piezoelectric constant of the GaN NW to be about $7.7 \mathrm{pm} / \mathrm{V}$ in [0001] crystallographic direction. The ultimate tensile strength of the GaN NW was obtained to be about 1.22 GPa. Our work demonstrates the power of in operando X-ray structural studies of single NWs for their effective design and implementation with desired functional properties.

28 KEYWORDS: GaN nanowires, coherent X-ray Bragg diffraction, piezoelectric effect, finite element method

29 emiconductor nanowires (NWs) based on gallium nitride

$30 \circlearrowleft(\mathrm{GaN})$, indium nitride $(\mathrm{InN})$, and indium gallium nitride

31 (InGaN) have promising applications for light-emitting diodes,

32 low-cost solar cells, transistors, single photon sources, and 33 other devices. ${ }^{1-6}$ The wurtzite (WZ) (hexagonal) crystal 34 structure of GaN NWs is noncentrosymmetric and has an 35 internal electric field along the [0001] crystallographic 36 direction. ${ }^{7,8}$ Local deformation of the GaN unit cell leads to 37 a formation of an internal piezoelectric field and vice versa. ${ }^{9}$ 38 Nitride-based light-emitting diodes (LEDs) grown on $c$-plane $39 \mathrm{GaN}$ substrate usually show the blue shifts with increased 40 injected current. This effect is usually attributed to the 41 screening of the piezoelectric field when the injected electron 42 density is high, which balances the band bending. It was also 43 demonstrated that a single GaN NW exhibits stronger 44 piezoelectricity $^{10}$ than a bulk GaN. ${ }^{11}$ Integration of the NWs 45 into an electric circuit by metallic contacts may induce 46 additional strain and, therefore, may lead to additional 47 piezoelectric effects in the structure. This may dramatically 48 influence electron-hole pair recombination and alter the 49 efficiency of optoelectronic devices based on GaN NWs. ${ }^{12}$
Moreover, better knowledge of the relation between piezo- 50 electric effect and strain field in single GaN NWs with the sizes 51 of hundreds of nanometers could potentially contribute to 52 understanding of inefficient recombination of electron-hole 53 pairs in quantum wells. ${ }^{13-15}$ Therefore, investigation of the 54 influence of applied voltages on the structure of a single $\mathrm{GaN} 55$ NW is of significant importance.

Different methods may be employed to reveal structural 57 changes in NWs under applied voltage such as scanning 58 electron microscopy (SEM) or transmission electron micros- 59 copy (TEM). ${ }^{16}$ X-ray nanodiffraction, developed recently at 60 synchrotron sources, is an alternative approach that allows one 61 to determine structural properties of single NWs in a 62 nondestructive way. ${ }^{17-20}$ Unfortunately, resolution of this 63 method is limited by the X-ray beam size. Newly developed X- 64 ray coherent scattering methods such as Bragg coherent X-ray 65 diffractive imaging (CXDI) and ptychography ${ }^{21-24}$ allow to 66

Received: May 3, 2018

Revised: July 17, 2018

Published: July 23, 2018 


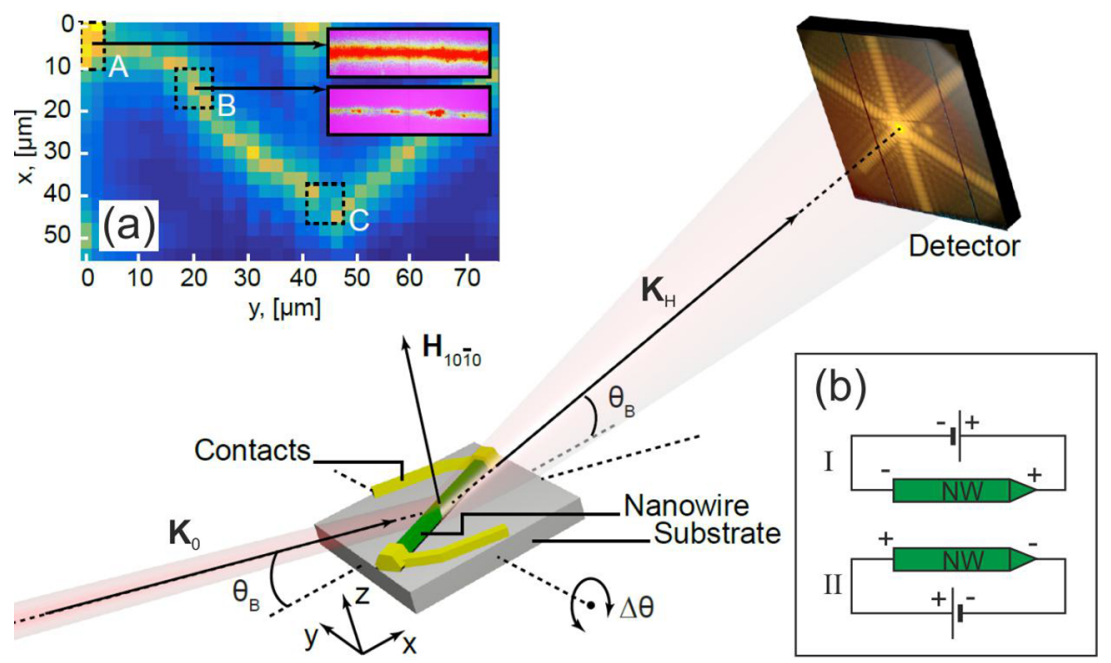

Figure 1. Scheme of the Bragg coherent scattering measurement geometry. The incoming X-ray beam $\mathrm{K}_{0}$ is scattered by a GaN NW at the Bragg angle $\theta_{\mathrm{B}}$ and resulting diffraction pattern is recorded by the $2 \mathrm{D}$ detector in the far-field. In the inset (a) the diffraction map of 111 Au Bragg reflection used for location of the NW is shown. Region A corresponds to the large Au bond pad; region B corresponds to the contacts deposited by lithography and thermal evaporation of $\mathrm{Ti}$ and $\mathrm{Au}$. Region $\mathrm{C}$ has a gap, where the GaN NW is located. The inset (b) schematically demonstrates two configurations of the NW connection to the power supply.

67 determine strain and deformation of single nanostructures with 68 the spatial resolution reaching $10 \mathrm{~nm}^{25-30}$ Moreover, these 69 techniques allow nondestructive investigation of nanostruc70 tures in situ and in operando. ${ }^{31}$ In this work, we applied 71 coherent X-ray Bragg diffraction to investigate the influence of 72 an applied voltage bias on the structure of a single GaN NW. 73 The samples were provided by the NanoLund Laboratory at 74 Lund University, Sweden. The GaN NWs with a diameter of $75350-400 \mathrm{~nm}$ and a length $3-4 \mu \mathrm{m}$ were grown on a $1 \mu \mathrm{m}$ 76 thick $\mathrm{GaN}$ template on top of a $\mathrm{Si}(111)$ substrate using metal 77 organic vapor phase epitaxy (MOVPE). ${ }^{32}$ The structure of 78 NWs was expected to be pure WZ without dislocations and 79 stacking faults. After the growth, GaN NWs were removed 80 from the original $\mathrm{GaN} / \mathrm{Si}$ substrate and deposited on a $\mathrm{Si}(111)$ 81 chip with a $100 \mathrm{~nm}$ thermally grown $\mathrm{SiO}_{2}$ layer on top, as an 82 insulating layer. Further, $220 \mathrm{~nm}$ thick metallic contacts were 83 deposited to connect the ends of single NWs to the pads using 84 electron beam lithography and thermal evaporation of $\mathrm{Ti}$ and $85 \mathrm{Au}$. First a $20 \mathrm{~nm}$ thick layer of $\mathrm{Ti}$ was deposited on $\mathrm{SiO}_{2}$ and ${ }_{86} \mathrm{GaN}$ NW to provide good adhesion between $\mathrm{Au}$ and $\mathrm{GaN}$, and 87 then $200 \mathrm{~nm}$ thick layer of $\mathrm{Au}$ on top of $\mathrm{Ti}$ layer. The position 88 and orientation of the NWs with respect to the main contacts 89 on the substrate could not be controlled during their 90 deposition. We implemented a sample holder, which provided 91 the electrical connections to the selected single NWs (see 92 Supporting Information). Electric measurements prior to the 93 X-ray experiment revealed the resistance of the NWs with 94 contacts to be about $10^{12} \mathrm{Ohm}$. Because of high resistivity, the 95 current through the NWs was on the order of a picoampere, 96 and additional cooling of the sample was not necessary.

97 The experiment was performed at the coherence beamline 98 P10 at the PETRA III synchrotron facility (DESY, Hamburg, 99 Germany). The geometry of the experiment is presented in 100 Figure 1. The X-ray beam with photon energy of $9.6 \mathrm{keV}$ and 101 flux of about $10^{11} \mathrm{ph} / \mathrm{s}$ was focused at the sample down to one 102 micrometer in size at full width at half-maximum (fwhm) using 103 compound refractive lenses. Characterization of the focus was 104 performed by knife edge scan at the sample position. The 105 measurements were performed at the six circle diffractometer.
It was equipped with a two-dimensional (2D) X-ray pixel 106 detector Lambda (pixel size of $55 \times 55 \mu \mathrm{m}^{2}$ ) positioned at a 107 distance of $1.96 \mathrm{~m}$ from the sample in Bragg geometry. An 108 evacuated flight tube was mounted between the sample and 109 detector to reduce air scattering. The sample with GaN NWs 110 was mounted on the diffractometer using an adapter with wires 111 connected to a power supply providing bias voltage (see 112 Supporting Information). The experimental setup allowed 113 measurements of $10 \overline{1} 0$ Bragg reflection of $\mathrm{GaN}$ with the 114 reciprocal lattice vector $\mathbf{H}_{10 \overline{1}}$ being normal to the substrate 115 (see Figure 1).

116

First, a free lying NW without Au electrodes was 117 investigated using 3D Bragg CXDI as a reference measurement 118 for further comparison with the NW under applied voltage. 119 After the X-ray beam was positioned at the center of the NW, 120 the rocking curve scan was performed in the angular range of 121 $\pm 0.4^{\circ}$ in the vicinity of the Bragg angle with 160 angular steps. 122 At each angular position diffraction patterns were recorded 123 with an exposure time of $20 \mathrm{~s}$.

The $3 \mathrm{D}$ intensity distribution around $10 \overline{1} 0 \mathrm{GaN}$ Bragg peak 125 for the free lying nanowire is shown in Figure 2 a (see SEM $126 \mathrm{f} 2$ images of this NW in the inset in Figure 2b). The isosurface of 127 intensity distribution around the Bragg reflection demonstrates 128 almost perfect hexagonal symmetry with the fringes originating 129 from the opposite facets of the free lying NW. The maximum 130 resolution achieved in the measurement was estimated to be 131 about $12 \mathrm{~nm}$ from the maximum $Q$-value reached in 3D 132 reciprocal space. The CXDI reconstruction of the reference 133 NW is presented in Figure 2b (see Supporting Information). 134 The reconstructed object has a hexagonal shape with the 135 distance between opposite side facets about $350 \mathrm{~nm}$ which is in 136 a good agreement with the SEM data. Additionally, after the X- 137 ray experiment, the same GaN NW was studied by the selected 138 area electron beam diffraction (see Figure 2c). The dark-field 139 transmission electron microscopy (DF-TEM) of $\overline{4} 131$ Bragg 140 diffraction of the GaN NW, which is originating only from WZ ${ }_{141}$ $\mathrm{GaN}$ crystal structure (see Figure 2d), confirmed absence of 142 defects and presence of only wurtzite phase in the GaN NW 143 (see Supporting Information). 


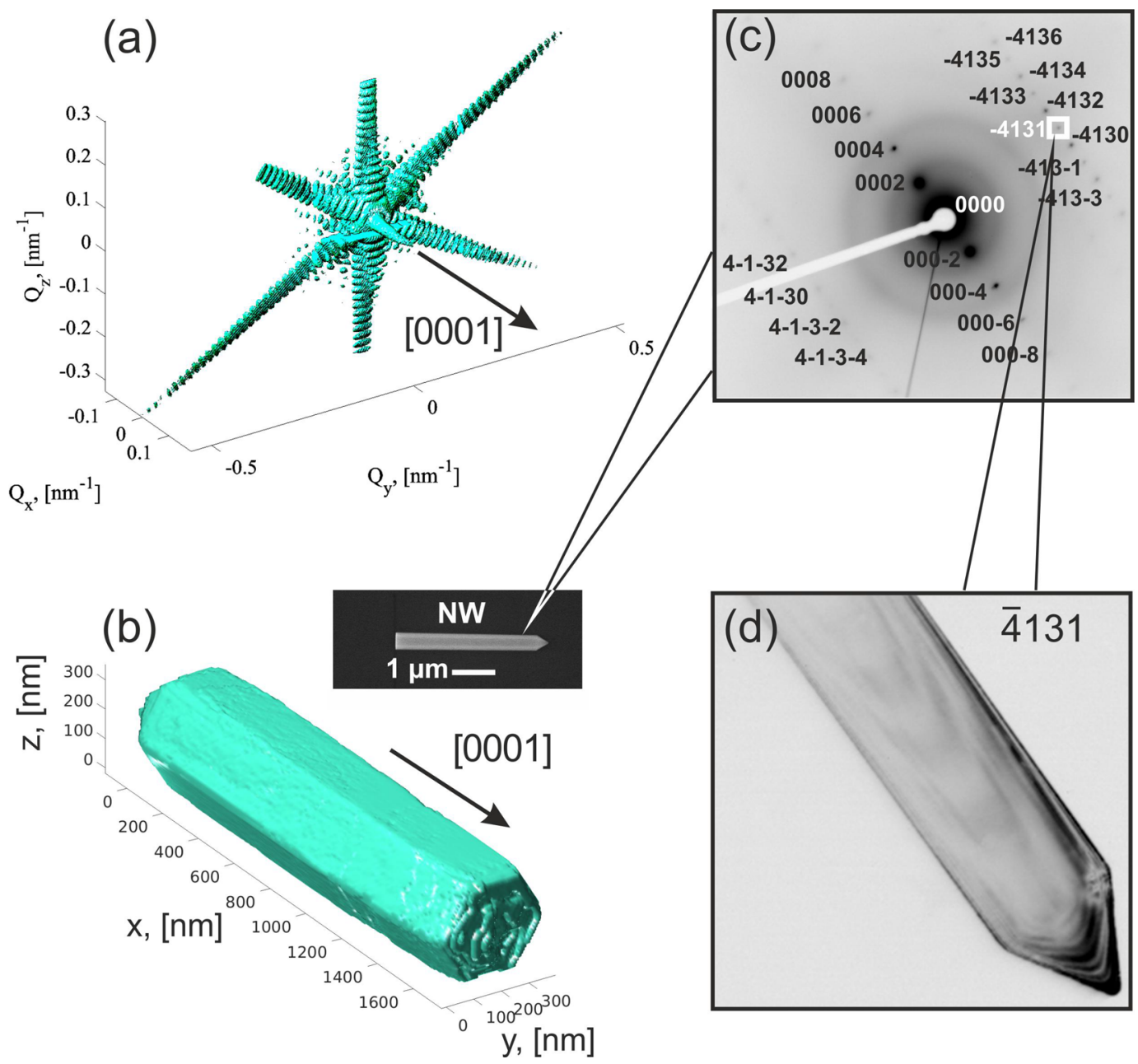

Figure 2. Study of the GaN NW without contacts. (a) Isosurface of the $3 \mathrm{D}$ intensity distributions around $10 \overline{10}$ Bragg reflection of GaN. The isosurface is plotted at $20 \%$ of the maximum value of the normalized logarithmic scale. The [0001] crystallographic direction is shown by an arrow. (b) Isosurface of the amplitude of the reconstructed NW. The corresponding SEM image of the studied NW is shown in the inset. (c) Electron beam diffraction on the same GaN NW performed after the X-ray experiment. Bragg reflections are indexed and direction [0000] corresponds to the direct electron beam. (d) Dark-field transmission electron microscopy (DF-TEM) image of the GaN NW for the $\overline{4} 131$ Bragg diffraction. The DF-TEM image demonstrates the absence of defects and confirms the presence of only wurtzite phase in the GaN NW.

145 Next, GaN NWs with deposited metal contacts were 146 investigated (see SEM image in the inset in Figure 3a). In 147 order to find such NWs, diffraction mapping of gold with the 148 step size of $1 \mu \mathrm{m}$ was performed in Bragg conditions of $111 \mathrm{Au}$ 149 reflection $\left(\theta_{\mathrm{B}}=15.92^{\circ}\right)$. Position of the selected NW was 150 identified as a gap in a gold signal (see inset a in Figure 1). 151 After the NW was located, the geometry of the setup was 152 changed to the Bragg conditions of a $10 \overline{10} \mathrm{GaN}$ reflection $\left(\theta_{\mathrm{B}}\right.$ $\left.153=13.54^{\circ}\right)$. This procedure was repeated for each NW with 154 metal contacts.

155 The $3 \mathrm{D}$ reciprocal space maps in the vicinity of the Bragg 156 angle were recorded by rocking the sample around $y$-axis and 157 recording far-field diffraction patterns on the $2 \mathrm{D}$ detector. The 158 angular range of the rocking scan for the NW with deposited 159 metal contacts was extended to $\pm 0.5^{\circ}$ around Bragg peak 160 position and 200 equally separated angular steps were 161 measured. An isosurface of the corresponding 3D intensity 162 distribution is presented in Figure $3 \mathrm{a}$ and reveals similar 163 hexagonal symmetry as in the case of the free lying NW. 164 However, the intensity of fringes was lower, decaying faster, 165 and slightly disturbed in comparison to the intensity 166 distribution of the reference free-lying NW (compare Figure
$3 \mathrm{a}$ and Figure 2a). At the same time, we observed new features 167 of the scattered intensity. The intensity distribution from the 168 NW with the deposited contacts consisted of two, not equally 169 intense, 6-fold "stars" with the separation between them along 170 the [0001] direction of the NW. We will call further the more 171 intense "star" of the Bragg peak as peak I and the second one 172 with lower intensity as peak II (see Figure 3). Similar features 173 were recently reported in refs 33 and 34 where nanodiffraction 174 studies of the single NWs were performed and were associated 175 with the bending of NWs. We also assign the peaks separation 176 in reciprocal space to scattering from different parts of the 177 GaN NW, caused by the NW bending (Figure 4a). Because no $178 \mathrm{f} 4$ chemical interaction or diffusion between $\mathrm{Au}$ and $\mathrm{GaN}$ is 179 expected, ${ }^{35}$ the observed deformation of the NW may be 180 predominantly attributed to the metal contacts depositing 181 process.

Further, the coherent scattering intensity distribution from 183 several GaN NWs with deposited metal contacts were 184 investigated as a function of the applied voltage bias. First, 185 we applied the positive polarity to the tip of the GaN NW in 186 [0001] direction (see configuration $\mathrm{I}$ in the inset $\mathrm{b}$ of Figure 187 1). In this case, the GaN lattice shrinks due to the piezoelectric 188 

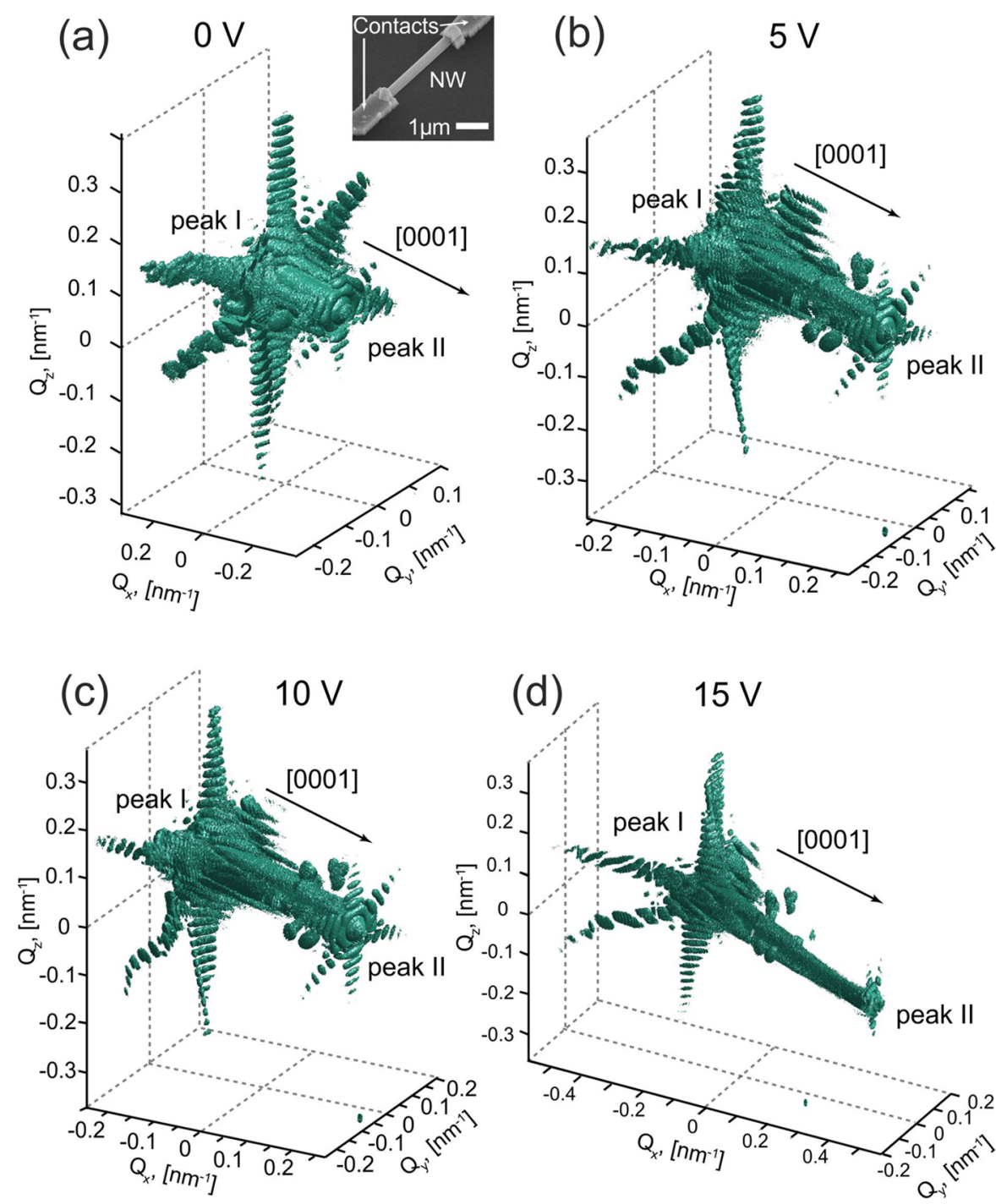

Figure 3. Isosurface of the 3D intensity distribution around $10 \overline{1} 0$ Bragg reflection of GaN NW under applied voltage bias of $0 \mathrm{~V}$ (a), $5 \mathrm{~V}(\mathrm{~b}), 10 \mathrm{~V}$ (c), and $15 \mathrm{~V}(\mathrm{~d})$. The intensity is presented in logarithmic scale and the isosurface values are taken at $20 \%$ of the maximum intensity value. The [0001] crystallographic direction is indicated by an arrow and two separated Bragg peaks, peak I and peak II, are depicted in the plots.
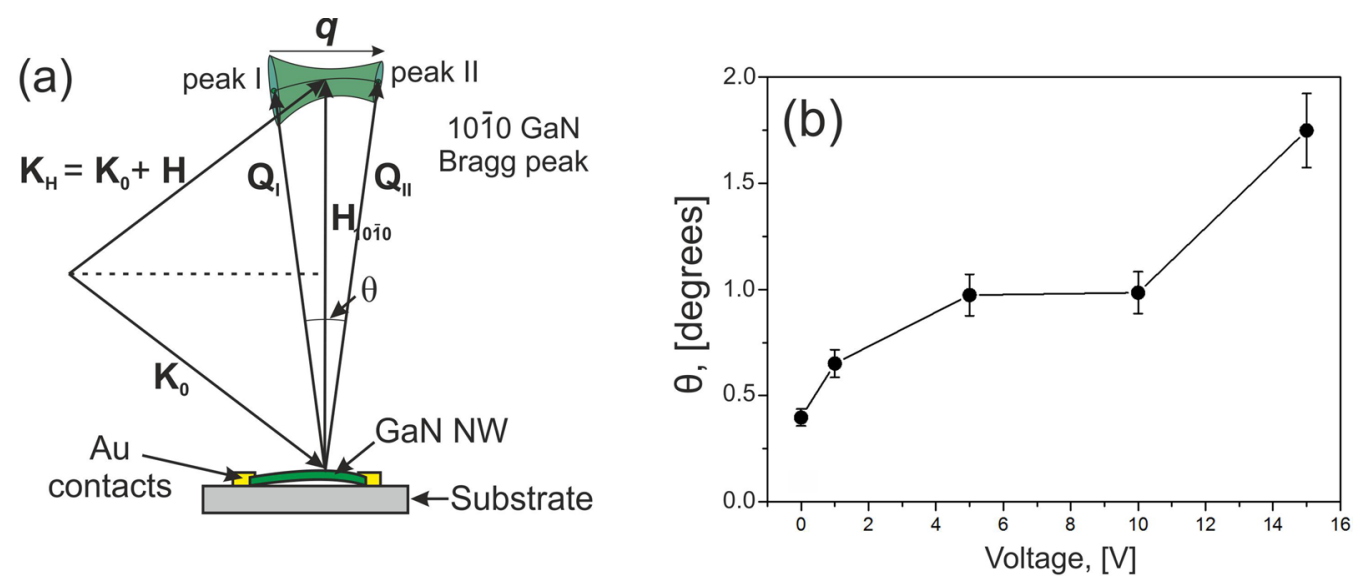

Figure 4. Schematics of X-ray scattering on a bent NW and bending angle $\theta$ dependence on the applied voltage bias. (a) Schematics of X-ray scattering on a bent NW. The reciprocal lattice vector $\mathbf{H}_{10 \overline{1} 0}$ is defined by the incident $\mathbf{K}_{0}$ and diffracted $\mathbf{K}_{\mathrm{H}}$ scattering vectors. The scattering vectors $\mathbf{Q}_{\mathrm{I}}$ and $\mathbf{Q}_{\mathrm{I}}$ of the Bragg peaks I and II correspond to the X-ray diffraction from different parts of the bent NW. Vector $\mathbf{q}$ defines separation between the scattering vectors $\mathbf{Q}_{\mathrm{I}}$ and $\mathbf{Q}_{\mathrm{I}}$ in reciprocal space. (b) Bending angle $\theta$ between the scattering vectors $\mathbf{Q}_{\mathrm{I}}$ and $\mathbf{Q}_{\mathrm{I}}$ as a function of the applied voltage bias. 

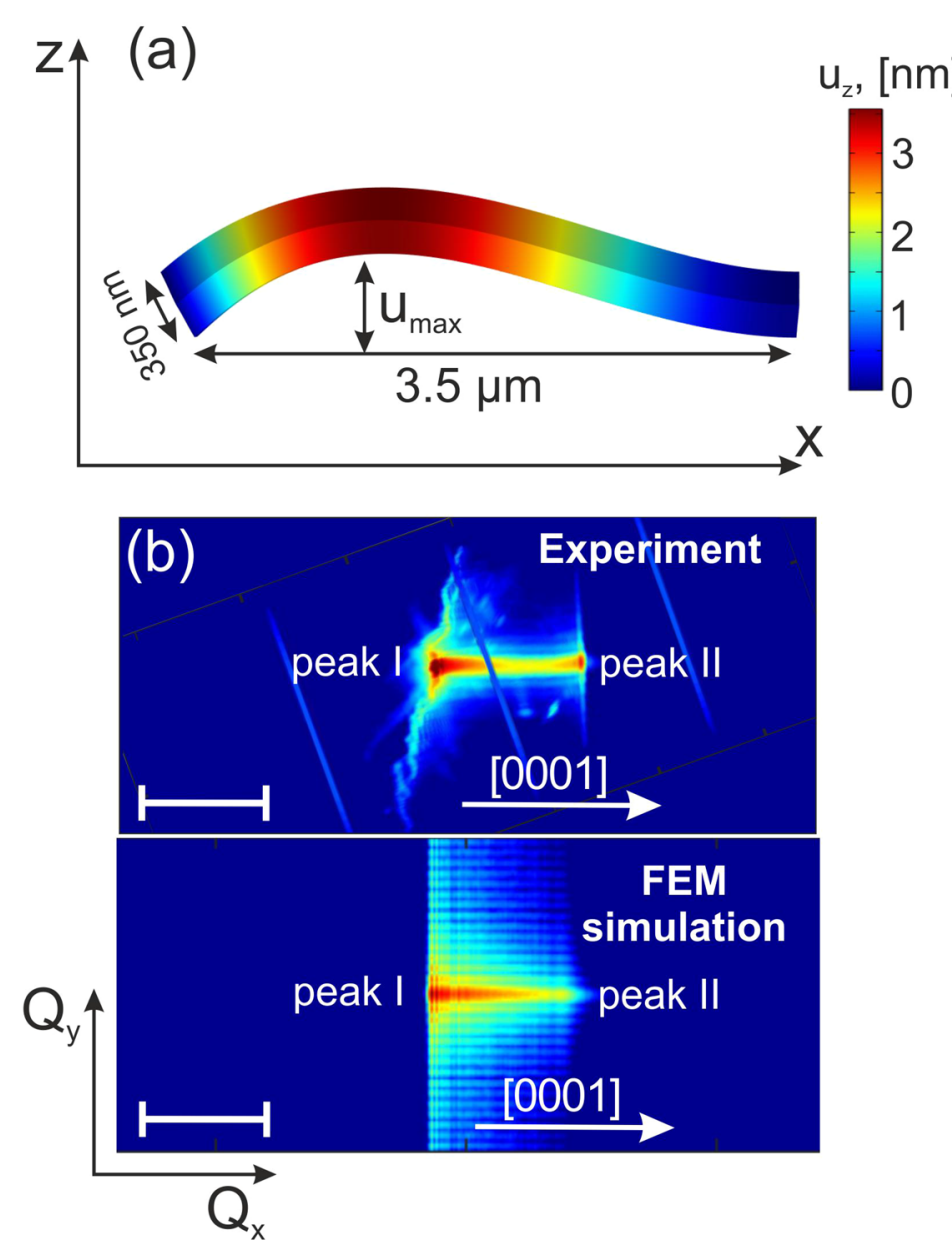
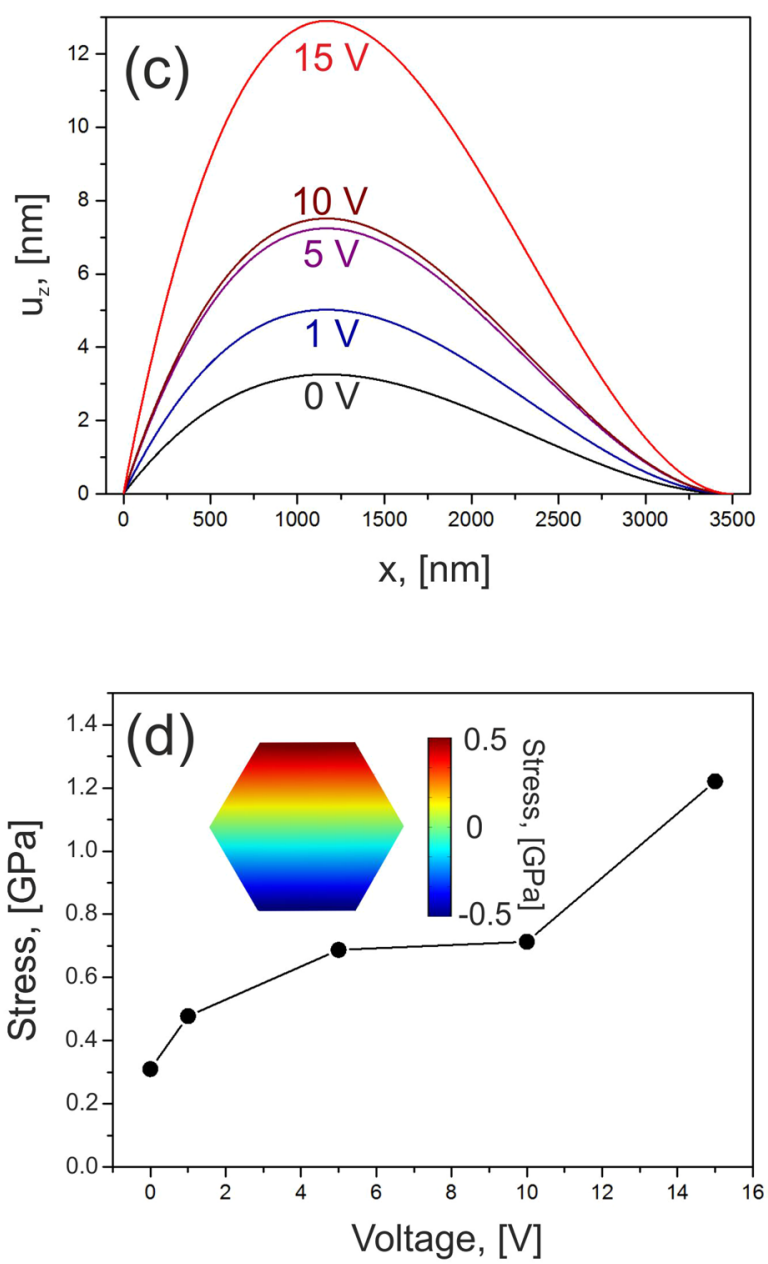

Figure 5. (a) Equilibrium shape of the NW with the displacement $u_{z}(x)$ determined from the analytical solution of the elasticity theory. Displacement in this figure is scaled 100 times for visibility. (b) Comparison of the experimental $10 \overline{1} 0 \mathrm{GaN}$ reciprocal space map corresponding to the voltage bias of $1 \mathrm{~V}$ (top) with the simulated one from the FEM model (bottom). The 3D scattered intensity of the GaN Bragg reflection was projected on $Q_{x} Q_{y}$ plane. The scale bars on the reciprocal space maps correspond to $0.2 \mathrm{~nm}^{-1}$. (c) Displacement profile $u_{z}(x)$ of the NW along its length determined by analytical solution and FEM model for different voltage values. (d) Dependence of the maximum value of the tensile stress in the slice of the NW at $x_{\max }=l / 3$ as a function of the applied voltage bias. The ultimate tensile strength of the GaN NW of 1.22 GPa was reached at the maximum voltage of $15 \mathrm{~V}$ after which the NW was broken. Stress values in a slice of the NW at the same position simulated by the FEM model for the voltage bias of $1 \mathrm{~V}$ is shown in inset. The positive values (red color) of stress are denoted to tension and negative (blue color) to compression of the GaN NW.

189 effect. Already at bias value of few volts the NW and gold were 190 disconnected due to the NWs contraction (see Supporting 191 Information). Next, we applied a negative polarity to the tip of 192 the GaN NW (see configuration II in the inset b in Figure 1). 193 As a result, the GaN lattice expanded in the [0001] 194 crystallographic direction. In this case, it was possible to 195 study evolution of the 3D Bragg peak intensity of the GaN NW 196 under applied voltage until its breakdown at $15 \mathrm{~V}$. Evolution of 197 the intensity distribution of the $10 \overline{1} 0 \mathrm{GaN}$ Bragg peak for the 198 applied voltage bias values of 5,10 , and $15 \mathrm{~V}$ is shown in 199 Figure $3 b-d$ (see Supporting Information). The damage of the $200 \mathrm{NW}$ was confirmed by the SEM studies at DESY NanoLab ${ }^{36}$ 201 after the X-ray scattering experiment (see Supporting 202 Information).

Direct reconstruction of the coherent scattering data shown 204 in Figure 3 by applying conventional reconstruction routines 205 turned out to be not feasible due to a large amount of strain 206 accumulated in the NWs with contacts and under applied 207 voltage bias (see Supporting Information).
In order to follow evolution of the intensity distribution of 208 the $10 \overline{1} 0 \mathrm{GaN}$ Bragg peak, we focus our attention on the 209 scattering vectors $\mathbf{Q}_{\mathrm{I}}$ and $\mathbf{Q}_{\mathrm{I}}$ of the corresponding peaks and 210 the relative distance between them defined by the vector $\mathbf{q}$ (see 211 Figure 4a). The modulus of the scattering vectors of the peaks $\mid 212$ $\mathrm{Q}$ is related to the $\mathrm{GaN}$ unit cell lattice parameter $a$ as 213 $|\mathbf{Q}|=2 \pi / d_{10 \overline{1} 0}=4 \pi / a \sqrt{3}$, where $d_{10 \overline{1} 0}$ is an interplanar distance between scattering planes corresponding to $10 \overline{1} 0214$ GaN Bragg peak. The calculated unit cell lattice parameters $a_{\mathrm{I}} 215$ and $a_{\mathrm{II}}$ of the peaks were found to be almost the same, 216 independent from the voltage bias, and equal to $a_{\mathrm{I}}=a_{\mathrm{II}}=217$ $3.1886 \pm 0.0003 \AA$ (see Supporting Information). This value 218 was by $1.33 \%$ larger than the lattice parameter of the free lying 219 $\mathrm{GaN} \mathrm{NW} a=3.1462 \pm 0.0003 \AA$ that means that the lattice of 220 the NW with contacts is expanding. The bending angle, 221 defined as $\theta \simeq|\mathbf{q}| /|\mathbf{H}|$, where $\mathbf{H}$ is the reciprocal space vector 222 of undistorted NW (see Figure 4a), was estimated to be about 223 $0.4^{\circ}$ without applied voltage for the GaN NW with metal 224 contacts and increased until $1.7^{\circ}$ when the voltage was 225 
226 changed to $15 \mathrm{~V}$. Its variation as a function of the applied 227 voltage is presented in Figure 4b.

228 Bending of the GaN NW under the applied voltage is the 229 major effect observed in our experiment. The NW was 230 modeled with its dimensions determined from the SEM 231 studies, such as, its size between opposite facets equal to $d=$ $232350 \mathrm{~nm}$ and length $l=3.5 \mu \mathrm{m}$. Because of its diameter-to233 length ratio (about 0.1 ) the $\mathrm{NW}$ can be considered as a thin 234 rod. We introduce the displacement field $u_{z}(x)$ in such a rod 235 which in our coordinated system is perpendicular to the 236 surface of the substrate and is along $z$-axis. According to the 237 elasticity theory, ${ }^{37}$ the displacement field in the absence of any 238 shear forces is defined by the differential equation $u_{z}^{\prime \prime \prime \prime}(x)=0$, 239 where the prime sign denotes derivative over the coordinate. 240 Solution of this equation with corresponding boundary 241 conditions is given by (see Supporting Information for details) $242 u_{z}(x)=u_{0}\left[(x / l)^{3}-2(x / l)^{2}+(x / l)\right]$, where $u_{0}=27 u_{\max } / 4$ and $243 u_{\max }$ is the maximum value of the function $u_{z}(x)$. From this 244 equation, we can directly determine that the maximum value $245 u_{\max }$ is located at the position $x_{\max }=l / 3$. Interestingly, this 246 position is in a good agreement with the length of the 247 remaining part of the broken NW determined by the SEM 248 measurements (see Supporting Information).

249 To relate now analytical solution of the bend NW with the 250 split of the Bragg peak observed in the experiment and caused 251 by this bending, we performed finite element method (FEM) 252 simulations. ${ }^{38}$ Material elastic properties and lattice constants 253 of the NW were obtained from corresponding GaN bulk 254 material values $^{9}$ (see Supporting Information). The equili255 brium shape of the NW used in FEM simulations and given by 256 the analytical solution discussed above is shown in Figure 5a. 257 The incident Gaussian beam profile with one micrometer 258 fwhm and 3D displacement field from the FEM model based 259 on analytical solution were used to simulate the intensity 260 distribution in reciprocal space, which was compared with the 261 experimental one (see Figure $5 \mathrm{~b}$ ). Varying parameter $u_{\max }$ in 262 the model, the best correspondence between the angular split 263 of the Bragg peaks in the model simulations and experimental 264 data were determined as it is shown in Figure $5 \mathrm{~b}$ for the $1 \mathrm{~V}$ 265 bias (see Supporting Information for other values of voltage). 266 Finally, the displacement field $u_{z}(x)$ of the NW was 267 determined for each applied voltage value and is shown in 268 Figure $5 \mathrm{c}$. As we see from this figure, after applying contacts to 269 the nanowire without any voltage already introduced a bend of 270 the NW with the maximum displacement value $u_{\max }=3.3 \mathrm{~nm}$. 271 By applying voltage, this maximum displacement was 272 substantially increased up to $u_{\max }=12.9 \mathrm{~nm}$ for the voltage 273 value $15 \mathrm{~V}$.

274 We used our analytical solution to the elasticity equation for 275 a thin rod to determine elongation $\Delta l$ of the NW as a function 276 of the applied voltage bias (see Supporting Information). By 277 that we found that the average value of the piezoelectric 278 constant $d_{33}$ of the GaN NW in [0001] crystallographic 279 direction defined by its elongation $\Delta l$ was about $d_{33}=7.7 \pm 2.2$ $280 \mathrm{pm} / \mathrm{V}$ (see Supporting Information). The determined value of 281 the piezoelectric constant of the GaN NW is lower than the 282 one measured previously for GaN NWs with sizes 64-191 nm 283 by piezoresponse force microscopy, ${ }^{10}$ which was about 12.8 $284 \mathrm{pm} / \mathrm{V}$, but is significantly higher than the bulk $\mathrm{GaN}$ value of 2 $285 \mathrm{pm} / \mathrm{V}^{11}$ (see for review ref 9 ).

286 Bending of the NW induces tensile and compressive strain 287 fields in the NW. ${ }^{37,39}$ As it was discussed above, we expect that 288 the failure of the GaN NW is more likely to start at the top facet of the NW at the position $x_{\max }=l / 3$ of the maximum 289 deformation value $u_{\max }$. Using the FEM model, stress values $\sigma 290$ in the slice of the NW at $x_{\max }$ were determined for all voltages. 291 Such slice for the voltage bias of $1 \mathrm{~V}$ is shown in the inset in 292 Figure $5 \mathrm{~d}$. The maximum tensile stress $\sigma_{\max }$ values on the top 293 facet of the NW were determined for different applied voltage 294 values and vary from $0.31 \mathrm{GPa}$ at $0 \mathrm{~V}$ to $1.22 \mathrm{GPa}$ at $15 \mathrm{~V}$ (see 295 Figure 5d). Because the mechanical fracture of the GaN NW in 296 our experiment occurred at $15 \mathrm{~V}$, the ultimate tensile strength 297 of the GaN NW was estimated to be about $1.22 \mathrm{GPa}$. 298

In summary, our measurements of the single GaN NWs with 299 and without deposited metal contacts demonstrated a visible 300 distortion of the $10 \overline{1} 0 \mathrm{GaN}$ Bragg peak intensity due to the 301 bend of the NW caused by the contacts. The bending angle 302 increased as a function of the applied voltage leading to 303 evolution of the diffraction pattern. Comparison of the 304 experimentally measured intensity distributions and the FEM 305 simulations allowed us to determine piezoelectric constant in 306 [0001] crystallographic direction with the value of $7.7 \mathrm{pm} / \mathrm{V} 307$ and ultimate tensile stress $\sigma_{\max }$ of about $1.22 \mathrm{GPa}$ that was the 308 origin of a mechanical break of the NW.

309

Our work demonstrates the power of in operando X-ray 310 structural studies of single NWs for their effective design and 311 implementation with desired properties. For our future 312 experiments, we plan to study the influence of the surface to 313 volume ratio of the NWs on their electro-mechanical 314 properties.

\section{ASSOCIATED CONTENT}

The Supporting Information is available free of charge on the 318 ACS Publications website at DOI: 10.1021/acs.nano- 319 lett. $8 \mathrm{~b} 01802$.

Description of the sample holder, reconstruction of the 321 free-lying GaN NW, transmission electron microscopy 322 of the free-lying GaN NW, SEM study of the GaN NWs 323 after the applied voltage bias, diffraction pattern of the 324 GaN NW evolution under applied voltage, analytical 325 calculation of a thin rod bending, FEM simulation 326 (PDF)

\section{AUTHOR INFORMATION}

\section{Corresponding Author}

*E-mail: ivan.vartaniants@desy.de.

Dmitry Dzhigaev: 0000-0001-8398-9480

Zhaoxia Bi: 0000-0002-7024-7266

Ivan A. Vartanyants: 0000-0002-0340-8234

Present Addresses

${ }^{\perp}$ (O.Y.G.) Department of Materials Science and Engineering, ${ }_{336}$ Cornell University, Ithaca, NY, 14850 United States.

\#(A.V.Z) European XFEL, Holzkoppel 4, 22869 Schenefeld, ${ }_{338}$ Germany.

Notes

The authors declare no competing financial interest.

\section{ACKNOWLEDGMENTS}

We would like to acknowledge Edgar Weckert for discussions 343 and support of the project, Tobias Spitzbart, Christopher Otte 344 and Lars Wilke from FS-EC department of DESY are 345 acknowledged for their help in the sample holder construction. 346 
$347 \mathrm{We}$ also acknowledge discussions on elasticity theory with 348 Vladimir Kaganer and careful reading of the manuscript by 349 Thomas Keller.

\section{$350 \square$ REFERENCES}

351 (1) Li, C.; Wright, J. B.; Liu, S.; Lu, P.; Figiel, J. J.; Leung, B.; Chow, 352 W. W.; Brener, I.; Koleske, D. D.; Luk, T.-S.; Feezell, D. F.; Brueck, S. 353 R. J.; Wang, G. T. Nano Lett. 2017, 17, 1049-1055.

354 (2) Lu, W. Semiconductor Nanowires: From Next-Generation 355 Electronics to Sustainable Energy; The Royal Society of Chemistry, 3562014.

357 (3) Feng, Z. C. III-Nitride Materials, Devices and Nano-Structures; 358 World Scientific Publishing Company Pte Limited, 2017.

359 (4) Dasgupta, N. P.; Sun, J.; Liu, C.; Brittman, S.; Andrews, S. C.; 360 Lim, J.; Gao, H.; Yan, R.; Yang, P. Adv. Mater. 2014, 26, 2137-2184. 361 (5) Kuykendall, T.; Ulrich, P.; Aloni, S.; Yang, P. Nat. Mater. 2007, $3626,951-956$.

363 (6) Huang, C.-T.; Song, J.; Tsai, C.-M.; Lee, W.-F.; Lien, D.-H.; 364 Gao, Z.; Hao, Y.; Chen, L.-J.; Wang, Z. L. Adv. Mater. 2010, 22, $3654008-4013$.

366 (7) Speck, J. S.; Chichibu, S. F. MRS Bull. 2009, 34, 304-312.

367 (8) Boxberg, F.; Søndergaard, N.; Xu, H. Q. Nano Lett. 2010, 10, $3681108-1112$.

369 (9) Espinosa, H. D.; Bernal, R. A.; Minary-Jolandan, M. Adv. Mater. 370 2012, 24, 4656-4675.

371 (10) Minary-Jolandan, M.; Bernal, R. A.; Kuljanishvili, I.; Parpoil, V.; 372 Espinosa, H. D. Nano Lett. 2012, 12, 970-976.

373 (11) Muensit, S.; Guy, I. L. Appl. Phys. Lett. 1998, 72, 1896-1898.

374 (12) El Kacimi, A.; Pauliac-Vaujour, E.; Eymery, J. ACS Appl. Mater. 375 Interfaces 2018, 10, 4794-4800.

376 (13) Zhao, Y.; Fu, H.; Wang, G. T.; Nakamura, S. Adv. Opt. 377 Photonics 2018, 10, 246-308.

378 (14) Scholz, F. Semicond. Sci. Technol. 2012, 27, 024002.

379 (15) Speck, J. S.; Chichibu, S. F. MRS Bull. 2009, 34, 304-312.

380 (16) Huang, J. Y.; Zheng, H.; Mao, S. X.; Li, Q.; Wang, G. T. Nano 381 Lett. 2011, 11, 1618-1622.

382 (17) Stankevič, T.; Hilner, E.; Seiboth, F.; Ciechonski, R.; Vescovi, 383 G.; Kryliouk, O.; Johansson, U.; Samuelson, L.; Wellenreuther, G.; 384 Falkenberg, G.; Feidenhans'l, R.; Mikkelsen, A. ACS Nano 2015, 9, $3856978-6984$.

386 (18) Stankevič, T.; Dzhigaev, D.; Bi, Z.; Rose, M.; Shabalin, A.; 387 Reinhardt, J.; Mikkelsen, A.; Samuelson, L.; Falkenberg, G.; 388 Vartanyants, I. A.; Feidenhans'l, R. Appl. Phys. Lett. 2015, 107, 389103101.

390 (19) Schroth, P.; Jakob, J.; Feigl, L.; Mostafavi Kashani, S. M.; Vogel, 391 J.; Strempfer, J.; Keller, T. F.; Pietsch, U.; Baumbach, T. Nano Lett. 392 2018, 18, 101-108.

393 (20) Stankevič, T.; Dzhigaev, D.; Bi, Z.; Rose, M.; Shabalin, A.; 394 Reinhardt, J.; Mikkelsen, A.; Samuelson, L.; Falkenberg, G.; 395 Vartanyants, I. A.; Feidenhans'l, R. Proc. SPIE 2015, 9592, 396 95920D-7.

397 (21) Robinson, I. K.; Harder, R. Nat. Mater. 2009, 8, 291-298.

398 (22) Rodenburg, J. M.; Hurst, A. C.; Cullis, A. G.; Dobson, B. R.; 399 Pfeiffer, F.; Bunk, O.; David, C.; Jefimovs, K.; Johnson, I. Phys. Rev. 400 Lett. 2007, 98, 034801.

401 (23) Nugent, K. Adv. Phys. 2010, 59, 1-99.

402 (24) Vartanyants, I. A.; Yefanov, O. M. X-ray Diffraction. Modern 403 Experimental Techniques. Coherent X-ray Diffraction Imaging of 404 Nanostructures; Pan Stanford Publishing: Singapore, 2015; Chapter 405 12, pp 341-384.

406 (25) Newton, M. C.; Leake, S. J.; Harder, R.; Robinson, I. K. Nat. 407 Mater. 2010, 9, 120-124.

408 (26) Godard, P.; Carbone, G.; Allain, M.; Mastropietro, F.; Chen, 409 G.; Capello, L.; Diaz, A.; Metzger, T. H.; Stangl, J.; Chamard, V. Nat. 410 Commun. 2011, 2, 568.

411 (27) Hruszkewycz, S. O.; Holt, M. V.; Murray, C. E.; Bruley, J.; Holt, 412 J.; Tripathi, A.; Shpyrko, O. G.; McNulty, I.; Highland, M. J.; Fuoss, 413 P. H. Nano Lett. 2012, 12, 5148-5154.
(28) Dzhigaev, D.; et al. J. Opt. 2016, 18, 064007.

(29) Dzhigaev, D.; Stankevic, T. Bi, Z, Lazarev, S, Rose, M.; 414 Shabalin, A.; Reinhardt, J.; Mikkelsen, A.; Samuelson, L.; Falkenberg, 416 G.; Feidenhans'l, R.; Vartanyants, I. A. ACS Nano 2017, 11, 6605- 417 6611.

(30) Hill, M. O.; et al. Nano Lett. 2018, 18, 811-819.

(31) Ulvestad, A.; Singer, A.; Cho, H.-M.; Clark, J. N.; Harder, R.; 420 Maser, J.; Meng, Y. S.; Shpyrko, O. G. Nano Lett. 2014, 14, 5123- 421 5127.

(32) Hersee, S. D.; Sun, X.; Wang, X. Nano Lett. 2006, 6, 1808- 423 1811. 424

(33) Wallentin, J.; Osterhoff, M.; Salditt, T. Adv. Mater. 2016, 28, 425 $1788-1792$.

(34) Wallentin, J.; Jacobsson, D.; Osterhoff, M.; Borgstroem, M. T.; 427 Salditt, T. Nano Lett. 2017, 17, 4143-4150.

(35) Sporken, R.; Silien, C.; Malengreau, F.; Grigorov, K.; Caudano, 429 R.; Sánchez, F.; Calleja, E.; Muñoz, E.; Beaumont, B.; Gibart, P.; et al. 430 MRS Internet J. Nitride Semicond. Res. 1997, 2, e23. 431

(36) Stierle, A.; et al. JLSRF 2016, 2, A76.

(37) Landau, L. D.; Lifshitz, E. M. Theory of Elasticity: Vol. 7 (Course 433 of Theoretical Physics), 3rd ed.; Elsevier, 2012.

(38) Reddy, J. N. An Introduction to the Finite Element Method; Asia 435 Higher Education Engineering/Computer Science Mechanical En- 436 gineering, 2005.

(39) Gere, J. M.; Timoshenko, S. P. Mechanics of materials; Boston 438 PWS Pub Co., 1997.

\section{8} .

\section{.}

